\title{
BMJ Open Systematic review of depressive, anxiety and post-traumatic stress symptoms among Asian American breast cancer survivors
}

William Tsai (D) , Sumaiya Nusrath, Ruidi Zhu

To cite: Tsai W, Nusrath S, Zhu R. Systematic review of depressive, anxiety and posttraumatic stress symptoms among Asian American breast cancer survivors. BMJ Open 2020;10:e037078. doi:10.1136/ bmjopen-2020-037078

- Prepublication history and additional material for this paper is available online. To view these files, please visit the journal online (http://dx.doi.org/10. 1136/bmjopen-2020-037078).

Received 17 January 2020 Revised 27 July 2020 Accepted 29 July 2020

A) Check for updates

(c) Author(s) (or their employer(s)) 2020. Re-use permitted under CC BY-NC. No commercial re-use. See rights and permissions. Published by BMJ.

Applied Psychology, New York University, New York, New York, USA

Correspondence to

Dr William Tsai;

will.tsai@nyu.edu

\section{ABSTRACT}

Objectives This paper aimed to review the experience of psychopathology symptoms (ie, depressive, anxiety and post-traumatic stress) and their social, cognitive and affective correlates among Asian American breast cancer survivors. Studies on psychosocial interventions for reducing psychopathology symptoms were also included in this review. Design A systematic review was conducted.

Methods PubMed, PsycINFO and Web of Science were searched from database inception to November 2018. Empirical, peer-reviewed articles on adult women of Asian heritage residing in the USA with breast cancer diagnoses were included in this review. The methodological quality of the included articles was coded.

Results The search yielded 16 empirical articles, which were all deemed to be of high methodological quality. Eleven studies utilised a quantitative design, two studies utilised a qualitative design and three studies utilised a mixed-methods design. Thirteen were cross-sectional and three were longitudinal in design. Only two intervention studies were identified. Studies showed that Asian American breast cancer survivors endorsed moderate to high levels of depressive symptoms, anxiety and post-traumatic stress symptoms; those who are more acculturated demonstrated lower levels of depressive and anxiety symptoms. Asian American breast cancer survivors with social constraints were more likely to have intrusive thoughts and, in turn, have high levels of psychopathology symptoms. Intervention studies were limited, but the use of community-based participatory research approaches and cultural adaptations were noted strengths of the studies.

Discussion In addition to discussing clinical implications, we highlight limitations of the literature, including a lack of longitudinal studies and the limited use of standardised diagnostic instruments for assessing psychopathology symptoms among this population. Clarifying the prospective relationships between psychopathology symptoms and their social, cognitive and affective correlates will help inform the development of culturally sensitive psychosocial interventions among Asian American breast cancer survivors.

\section{INTRODUCTION}

Breast cancer is the most common type of cancer and the second leading cause of cancer death among Asian American women. ${ }^{1}$ Asian Americans are the fastest

\section{Strengths and limitations of this study}

- Sixteen articles were included in the review after searching PubMed, PsycINFO and Web of Science databases.

- The systematic review protocol was registered on the PROSPERO International Prospective Register of Systematic Review.

- Despite the limited number of studies, all included studies were of high methodological quality.

- The review included empirical studies utilising a variety of designs, including quantitative (crosssectional, longitudinal), qualitative (structured/semistructured interviews, in-depth interviews, focus groups) and mixed-methods study designs.

growing group in the USA that is projected to double in size by $2060 .^{2}$ Compared with the breast cancer incidence rates that have stabilised for other racial groups in the USA, the breast cancer incidence rate for Asian American women has steadily increased over the past two decades. ${ }^{3}{ }^{4}$ With the prognosis for breast cancer continuing to improve, ${ }^{5}$ a sizeable number of Asian American breast cancer survivors is expected in the future. To our knowledge, only one systematic review of Asian American breast cancer survivorship experiences exists in the literature. ${ }^{6}$ However, Wen and colleagues ${ }^{6}$ focused on healthrelated quality of life as the main outcome of interest, and findings focused on psychopathology (eg, depression, anxiety and posttraumatic stress) have yet to be synthesised. A focus on psychopathology is warranted as there is considerable evidence that rates of depression and suicide are higher among cancer survivors than among healthy controls. ${ }^{7-9}$

The examination of depression, anxiety and post-traumatic stress among Asian American breast cancer survivors is dispersed sparsely in the literature. The lack of investigation on the 
role of culture, in particular, inhibits a full understanding of the psychopathology symptoms experienced by Asian American breast cancer survivors. Asian American breast cancer survivors may hold culturally specific beliefs about cancer and adhere to cultural norms related to supportseeking and emotional disclosure that have been linked to cancer adjustment. ${ }^{1011}$ Collectivistic values, which are a cultural set of values commonly held by Asian individuals that prioritise the well-being of in-group members over one's own, emphasise the importance of maintaining social harmony and fulfiling relational obligations with close family and friends. ${ }^{12} 13$ For example, Asian American breast cancer survivors may suppress cancer-related distress and avoid seeking support to save face or prevent their caregivers from experiencing excessive burden. ${ }^{11}$ Adherence to these cultural values and the presence of various socioecological barriers (eg, limited access to healthcare resources) are associated with psychological distress during cancer survivorship. For instance, Asian American breast cancer survivors who are less acculturated to mainstream American culture are more likely to experience a lower quality of life than their more acculturated counterparts. ${ }^{14-16}$

This review aims to consolidate the extant literature by answering the following three questions. First, what is the experience of psychopathology symptoms among Asian American breast cancer survivors and the role of acculturation? Second, what are the social, cognitive and affective correlates of psychopathology symptoms among this population? And finally, what psychosocial interventions are effective for reducing psychopathology symptoms among Asian American breast cancer survivors?

\section{METHODS}

This review observed appropriate Preferred Reporting Items for Systematic Reviews and Meta-Analyses and MOOSE guidelines ${ }^{17} 18$ and the protocol (PROSPERO 2018 CRD42018117235) was registered on the PROSPERO International Prospective Register of Systematic Review (https://www.crd.york.ac.uk/prospero/display_ record.php?RecordID=117235).

\section{Eligibility criteria}

Studies eligible for inclusion in this systematic review were empirical, peer-reviewed papers. Grey literature, such as dissertations, book chapters, conference abstracts and presentations, as well as systematic reviews and metaanalysis reviews, were excluded. The included studies must have enroled adult (18 years of age or above) women of Asian heritage living inside the USA with a diagnosis of breast cancer. Given the limited empirical data on Asian Americans with cancer diagnoses, we defined 'survivorship' as patients with cancer following diagnosis until the end of life. Additionally, all reports were written or available in the English language and utilised quantitative (cross-sectional, longitudinal), qualitative (structured/ semistructured interviews, in-depth interviews, focus groups) or mixed-methods study designs.

\section{Outcomes of interest}

Reports of original research were screened for the inclusion of psychopathology symptoms (eg, depressive symptoms, anxiety symptoms, post-traumatic stress symptoms).

\section{Theoretical framework}

In order to understand the social, cognitive and affective factors associated with psychopathology among Asian American breast cancer survivors, we used the social-cognitive processing (SCP) model as a framework for understanding the results. ${ }^{19}$ The SCP model connected positive and negative aspects of cancer survivors' external social environments with their internal cognitive and emotional processes related to cancer. ${ }^{19}$ The central tenant of the SCP model was that individuals who were constrained by their social environments experienced disruptions in their ability to cognitively and emotionally process their cancer experiences. These disruptions, in turn, may be associated with higher levels of psychopathology symptoms. ${ }^{20} 21$

\section{Search strategy}

The databases PubMed, PsycINFO and Web of Science were used to identify peer-reviewed articles published from database inception until November 2018. The search strategy employed a combination of three concepts corresponding to our outcomes of interest. Concept one: breast cancer; concept two: psychopathology, psychiatric symptoms, psychological problems, depressive symptoms, depression, anxious symptoms, anxiety and post-traumatic stress; and concept three: Asian, Asian American, Chinese, China, Korean, Korea, Japanese, Japan, Vietnamese, Vietnam, Filipino, Philippines, Malaysia, Malaysian, Pakistani, Pakistan, Thai, Thailand, Indian and India. For all searches conducted, please see online supplemental table S1.

After the initial database search, the two coauthors with backgrounds in counselling psychology independently screened the titles and abstracts to determine whether the full texts of the articles should be reviewed. The coauthors then reviewed the reference sections of these articles for additional eligible studies. Consensus was achieved after the authors discussed differences in their determination of article inclusion. The first author, with a background in clinical health psychology, was brought in to break any ties in consideration for article inclusion. The final included studies that met study criteria were coded by two authors to extract relevant data. The following information was gathered from each article included in the review: publication characteristics (eg, year of publication, author names), study characteristics (eg, study design, outcomes assessed) and sample characteristics (eg, breast cancer stage, mean age, sample location and size).

\section{Data quality assessment}

To assess the methodological quality of the included articles, a coding scheme (see table 1) was created based on 
Table 1 Criteria for assessing the methodological quality of included studies

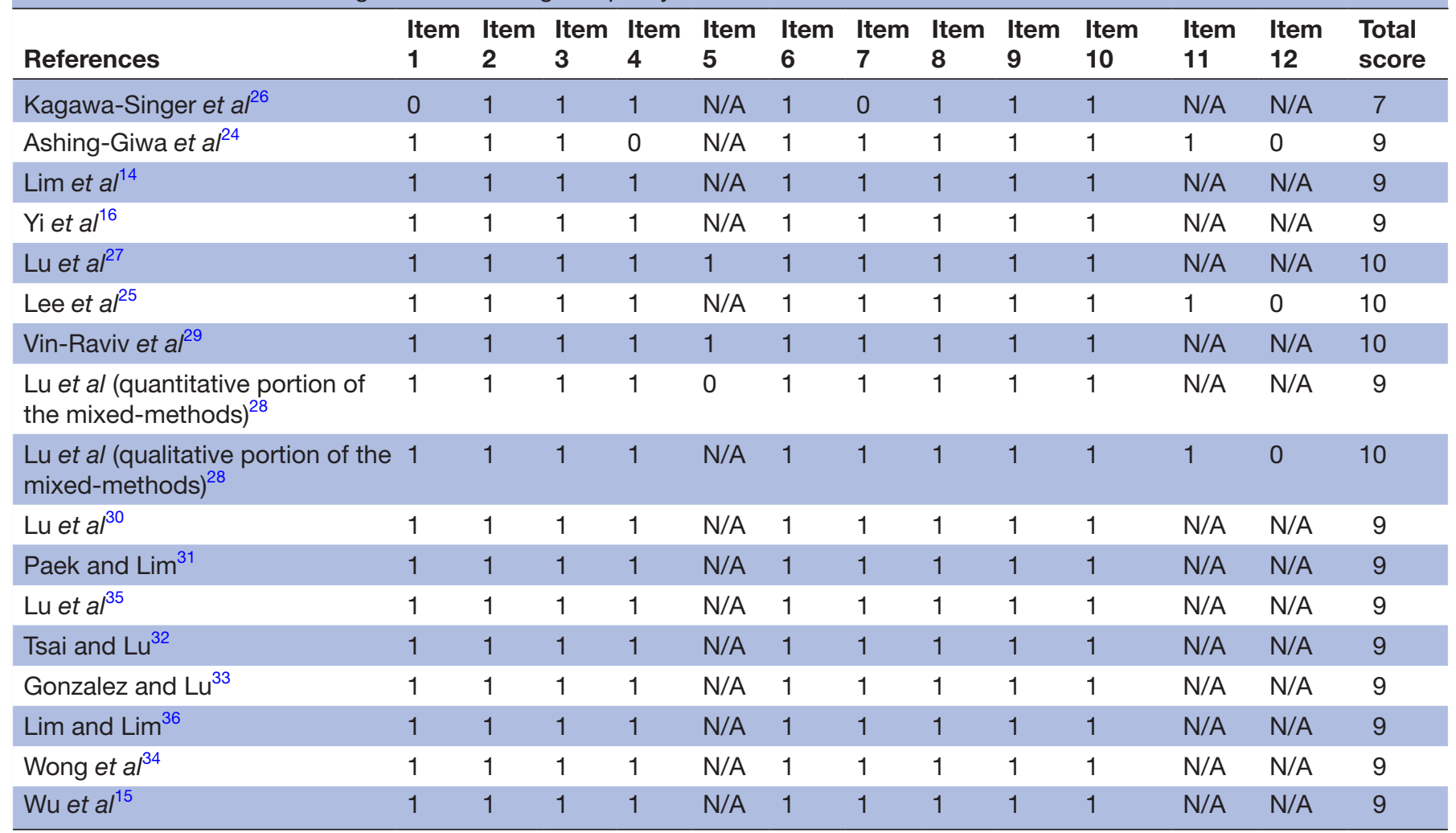

Methodological quality was calculated by assessing the following criteria for quantitative studies: (1) sociodemographic and clinical variables are described (eg, age, race, time since diagnosis, breast cancer stage); (2) inclusion and exclusion criteria are explained; (3) data collection methods (eg, interview, focus group, self-report) and study design (quantitative, qualitative, mixed-methods) are discussed; (4) psychopathology symptom is assessed using validated measures or interview question rationale is discussed; (5) if longitudinal in design: follow-up times and attrition rates are described; (6) study outcomes are clearly mentioned and appropriate/relevant measures are utilised; (7) results are presented with strengths and weaknesses, and clear distinctions are made between data and its interpretation; (8) appropriate analysis techniques are employed to report on main outcomes; (9) Institutional review board approval gained and participant consent obtained and (10) participant recruitment explained, location of research stated and the selection of cases or participants is theoretically justified. For qualitative studies, two additional criteria were assessed: (11) appropriate discussion of how themes, concepts and categories were derived from the data and (12) measures have been taken to test the validity of the findings.

previously established criteria for systematic reviews. ${ }^{22}$ One point was awarded for each study criteria if it was described sufficiently in the article. A quantitative study's highest possible score was 9 and for a cross-sectional study or a longitudinal study the highest possible score was 10 . Qualitative studies had a highest possible score of 11 . Studies scoring $75 \%$ or more of the maximum possible points were considered to be 'high quality,' with studies scoring between $50 \%$ and $75 \%$ rated as 'moderate quality' and studies scoring $<50 \%$ considered 'low quality'. ${ }^{23}$

\section{Patient and public involvement}

This research was done without patient involvement.

\section{RESULTS}

The initial database searches yielded 1164 articles after 4587 duplicates were removed. After screening the titles and abstracts of these 1164 articles, 35 articles were selected for a full-text review. From reviewing the reference sections of these 35 articles, 19 additional articles were retrieved for further full-text review, resulting in a total of 54 full-text articles that were independently assessed by two coauthors to determine eligibility for inclusion in this review. A total of 16 articles were ultimately included in this review (see figure 1), the earliest of which was published in 1997. Most studies $(n=9$; $56.25 \%$ ) were published between 2014 and 2018. With agreement from both coders, the results from the data quality assessment demonstrated that all 16 articles were coded as 'high quality' (see table 1).

\section{Characteristics of included studies}

Among the 16 included studies, 11 studies utilised a quantitative design, two studies utilised a qualitative design ${ }^{24} 25$ and three studies utilised a mixed-methods design. ${ }^{26-28}$ However, only one of the three mixed-methods design studies reported their qualitative findings. ${ }^{28}$ Among the included studies, 13 were cross-sectional and three were 


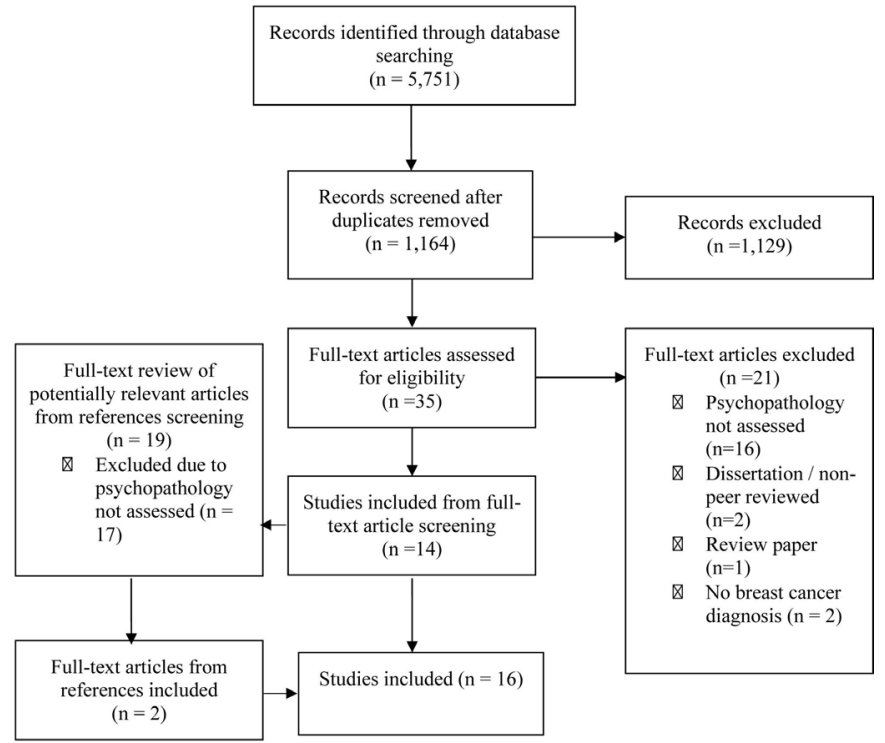

Figure 1 Process of literature search and review.

longitudinal in design. ${ }^{27-29}$ Of the 16 included studies, two intervention studies were identified. ${ }^{27} 28$ Seven studies focused solely on depressive symptoms, ${ }^{14} 26$ 30-34 four studies focused solely on post-traumatic stress symptoms ${ }^{152729} 35$ and one study focused solely on anxiety symptoms. ${ }^{24}$ Three studies examined both anxiety and depressive symptoms, ${ }^{162836}$ and only one study examined all three psychopathology symptom types. ${ }^{25}$ Ten studies focused their investigation on one Asian American subgroup (eg, Chinese American), while the rest of the studies collected data from multiple Asian American subgroups. ${ }^{16} 263136$ The identified studies mostly examined Chinese Americans ( $n=14)$, with fewer studies examining Korean Americans $(n=5)$ and Japanese Americans $(n=2)$. Three studies included cross-cultural comparisons between Asian and non-Asian groups. ${ }^{24} 2629$ While all studies commented on the role of culture in breast cancer adjustment, only six studies examined culture as a variable in their analyses (eg, acculturation level). ${ }^{14-16263233}$ Key study information and summary of findings are provided in tables 2 and 3 , respectively.

\section{Experience of depressive, anxiety and post-traumatic stress symptoms among Asian American breast cancer survivors and the role of acculturation}

One study with Chinese American, Japanese American and European American breast cancer survivors found that all ethnic groups experienced moderate levels of depressive symptoms. ${ }^{26}$ When Chinese American and Japanese American breast cancer survivors were compared on depressive symptoms in relation to acculturation, no significant difference along acculturation levels was found. However, these findings should be interpreted in light of small group sizes $(n=13,11$ and 11 for European American, Chinese American and Japanese American, respectively). In another quantitative study, both Chinese American and Korean American breast cancer survivors experienced moderate to high levels of depressive symptoms. ${ }^{31}$ However, conclusions regarding whether Asian Americans experience higher or similar levels of depressive symptoms cannot be drawn without the inclusion of other cultural groups.

Most studies included in this review examined the impact of breast cancer on depressive symptoms within only one Asian subgroup (eg, Chinese Americans). With the exception of one study, ${ }^{26}$ we found a consistent relationship between higher levels of acculturation and lower depressive symptoms. Higher levels of acculturation were associated with greater positive social networks and emotional support, which in turn was associated with lower depressive symptoms among a sample of Korean immigrant breast cancer survivors. ${ }^{14}$ Chinese American and Vietnamese American breast cancer survivors with greater English proficiency endorsed lower depressive and anxiety symptoms than their counterparts with lower English proficiency. ${ }^{16}$ Notably, Yi and colleagues ${ }^{16}$ conducted the only quantitative study that assessed anxiety symptoms among Vietnamese American and Chinese American breast cancer survivors among the 16 included studies.

In the only study that examined whether post-traumatic stress symptoms reached clinical levels, as well as the only non-intervention study that employed a longitudinal design in this review, Vin-Raviv and colleagues ${ }^{29}$ found that Asian American women were more likely to endorse clinical levels of post-traumatic stress (as indicated by a score of 24 or higher on the Impact of Event Scale $^{37}$ ) than European American women after receiving a breast cancer diagnosis. Almost $30 \%$ of the Asian American women sampled in this study endorsed clinical levels of post-traumatic stress symptoms, compared with only $21 \%$ of the European American women. Asian American women, relative to European American women, were also more likely to develop clinical levels of post-traumatic stress symptoms over a 6-month period.

\section{Social, cognitive and affective correlates of psychopathology} symptoms among Asian American breast cancer survivors

In a qualitative study using in-depth interviews, Korean American and Chinese American breast cancer survivors revealed difficulties with the open discussion of feelings despite experiencing high levels of depression and anxiety. ${ }^{25}$ Corroborating these findings, a Korean American breast cancer survivor shared, 'I wasn't afraid of death, but the pain...I didn't want to show myself screaming from pain to my family. ${ }^{24}$ The social constraints related to communication and emotional disclosure are not limited to interactions with close others. Chinese American breast cancer survivors endorsed communication dissatisfaction with their physicians, and the dissatisfaction was worse among Chinese American women with low acculturation. ${ }^{26}$ Three quantitative studies showed that social constraints and intrusive thoughts were associated with greater post-traumatic symptoms ${ }^{30} 35$ and depressive symptoms ${ }^{34}$ among Chinese American breast cancer survivors. In a cross-sectional path model with Chinese 


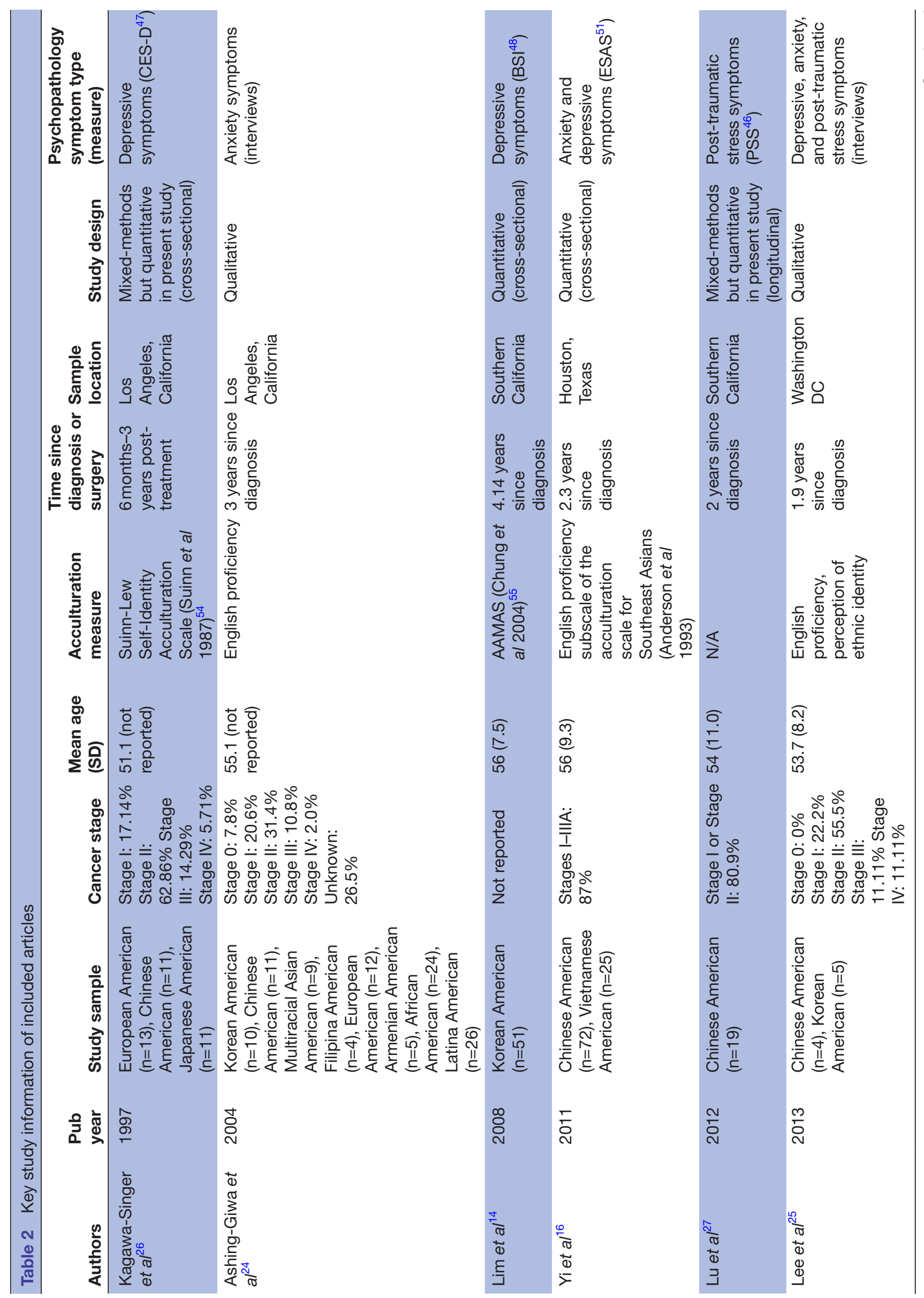




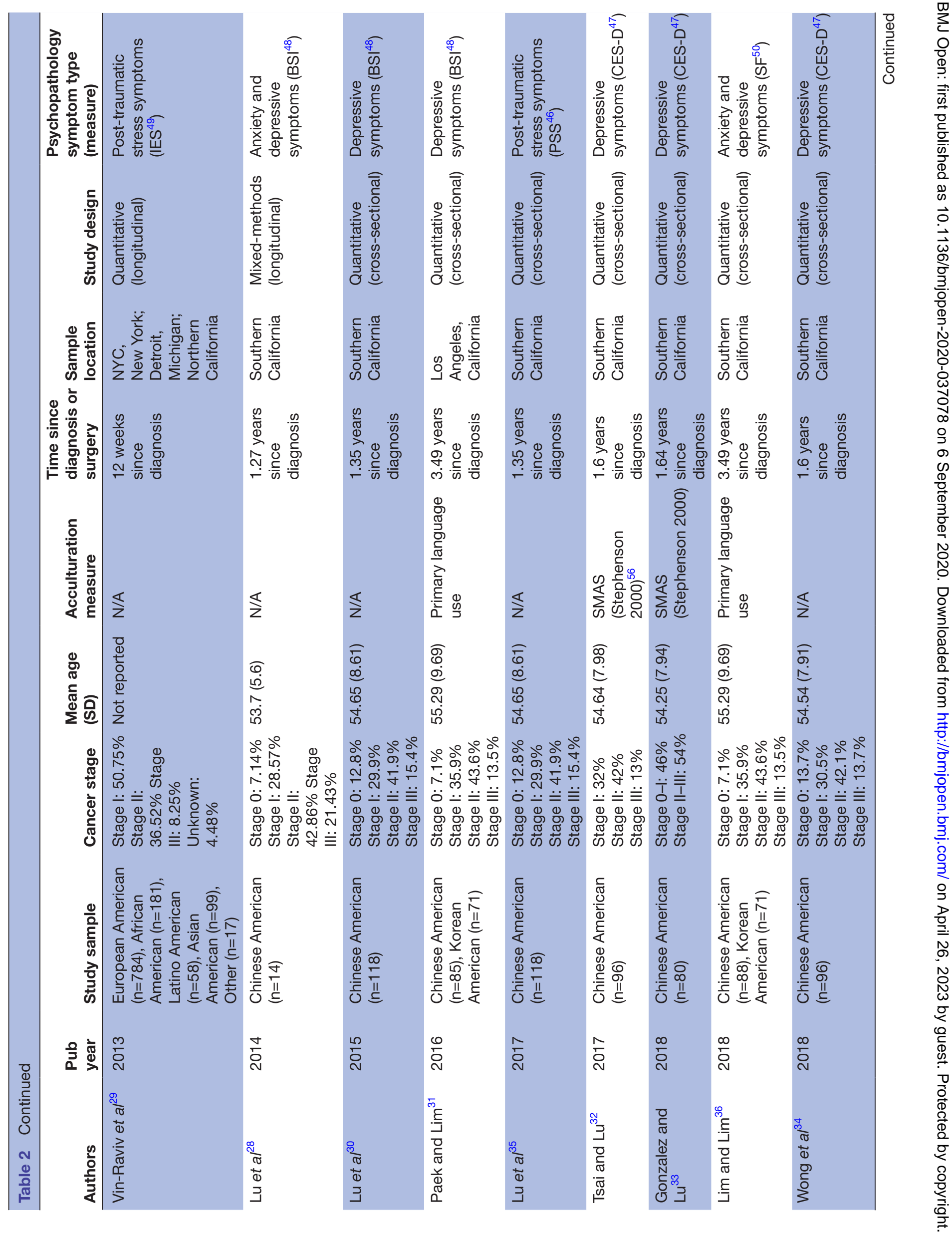


American breast cancer survivors, ${ }^{34}$ social constraints were associated with greater intrusive thoughts, lower social support and higher ambivalence over emotion expression (AEE). These psychological processes, in turn, were associated with greater depressive symptoms. ${ }^{34}$ The direct effect of social constraints on depressive symptoms was no longer significant after the mediators were included in the model.

AEE is another form of constraint that has been examined as a risk factor during survivorship. AEE is defined as the inner conflict between wanting to express emotions but also fearing the consequences of doing so. ${ }^{38}$ For example, Asian American breast cancer survivors with high levels of AEE may want to express their cancer-related distress, but fear adding burden to their caregivers. Across studies with Chinese American breast cancer survivors, AEE has been linked with greater depressive and post-traumatic stress symptoms. ${ }^{15} 303234$ However, the positive relationship between AEE and depressive symptoms was attenuated for those with lower levels of acculturation. ${ }^{32}$

\section{Psychosocial interventions for reducing psychopathology symptoms among Asian American breast cancer survivors}

The need for linguistically and culturally appropriate interventions and resources was frequently mentioned during in-depth interviews by Chinese American and Korean American cancer survivors. ${ }^{25}$ Although none of the interviewees attended a support group, some stated that they would be willing to attend if a linguistically appropriate one existed. The benefits of such support groups were echoed by the Asian American oncologists who were interviewed in this study.

Two psychosocial intervention studies were included in this review. ${ }^{27}{ }^{28}$ A small pilot study ( $\mathrm{n}=19$ Chinese American breast cancer survivors) found that the expressive writing intervention was associated with decreases in posttraumatic stress symptoms and intrusive thoughts at the 3-month follow-up. ${ }^{27}$ The second intervention study was a mixed-methods evaluation of a 10-week education and social support programme that recruited the help of peer mentors (ie, trained Chinese Americans who are breast cancer survivors themselves) to provide peer support for Chinese American breast cancer survivors. Results from the quantitative assessment and focus group discussions revealed that Chinese American breast cancer survivors who participated in this intervention experienced fewer depressive and anxiety symptoms. ${ }^{28}$

\section{DISCUSSION}

This systematic review was based on three research questions. The first question addresses the experience of common psychopathology symptoms (ie, depressive, anxiety and post-traumatic stress symptoms) among Asian American breast cancer survivors and the influence of acculturation. When comparing across cultural groups, Kagawa-Singer and colleagues ${ }^{26}$ found no ethnic group differences in the moderate levels of depressive 
Table 3 Main findings of included articles

\begin{tabular}{|c|c|}
\hline References & Main findings \\
\hline Kagawa-Singer et al ${ }^{26}$ & $\begin{array}{l}\text { No significant differences between Japanese Americans, Chinese Americans and European } \\
\text { Americans were found in depressive symptoms. Acculturation did not moderate the level of } \\
\text { depressive symptoms among the Asian American groups. }\end{array}$ \\
\hline Ashing-Giwa et $a l^{24}$ & $\begin{array}{l}\text { Across all ethnic groups (ie, African Americans, Asian Americans, European Americans and Latina } \\
\text { Americans), breast cancer survivors expressed similar anxiety regarding cancer recurrence, pain and } \\
\text { death. }\end{array}$ \\
\hline Yi et $a l^{16}$ & $\begin{array}{l}\text { Lower English proficiency was associated with higher anxiety and depressive symptoms among } \\
\text { Vietnamese and Chinese American breast cancer survivors. }\end{array}$ \\
\hline Lu et $a l^{27}$ & $\begin{array}{l}\text { Expressive writing was associated with long-term improvement in post-traumatic stress symptoms } \\
\text { among Chinese American breast cancer survivors. }\end{array}$ \\
\hline Vin-Raviv et $\mathrm{al}^{29}$ & $\begin{array}{l}\text { Nearly } 25 \% \text { of women diagnosed with breast cancer reported clinical levels of post-traumatic stress } \\
\text { symptoms, with increased risk among African American and Asian American women. }\end{array}$ \\
\hline Lu et $a l^{28}$ & $\begin{array}{l}\text { The peer-mentoring and education intervention programme was associated with lower depressive } \\
\text { symptoms among Chinese American breast cancer survivors. Focusing on relationship building may } \\
\text { be fruitful for designing novel interventions for cancer survivors from collectivistic cultures. }\end{array}$ \\
\hline Lu et $\left.a\right|^{30}$ & $\begin{array}{l}\text { Chinese American breast cancer survivors who were highly ambivalent over emotional expression } \\
\text { experienced higher levels of depressive symptoms. }\end{array}$ \\
\hline Paek and Lim ${ }^{31}$ & Family communication strain mediated the relationship between life stress and depressive symptoms. \\
\hline Lu et $a l^{27}$ & $\begin{array}{l}\text { Chinese American breast cancer survivors who were highly ambivalent about emotion expression had } \\
\text { higher post-traumatic stress symptoms. }\end{array}$ \\
\hline Wu et $a l^{15}$ & $\begin{array}{l}\text { Chinese American breast cancer survivors with higher ambivalence over emotion expression } \\
\text { experienced greater post-traumatic stress symptoms. Post-traumatic stress symptoms mediated the } \\
\text { relationship between ambivalence over emotion expression and physical functioning. The indirect } \\
\text { effects were stronger for those who were more acculturated to mainstream culture. }\end{array}$ \\
\hline
\end{tabular}

symptoms experienced by Chinese American, Japanese American, and European American breast cancer survivors. However, in another study, Asian American breast cancer survivors endorsed higher levels of post-traumatic stress symptoms than European American breast cancer survivors and were more likely to experience clinical levels of post-traumatic stress symptoms over time. ${ }^{29} \mathrm{~A}$ previous study with mostly European American breast cancer survivors found average Centre for Epidemiologic Studies Depression Scale (CES-D) depressive symptom scores of $8,{ }^{39}$ compared with average CES-D depressive symptom scores of 10.31 reported among Chinese American breast cancer survivors in Tsai and Lu. ${ }^{32}$ Similarly, the brief symptom inventory (BSI) depressive (5.4) and anxiety symptom scores (6) reported among Chinese
American breast cancer survivors in Lu and colleagues ${ }^{28}$ are markedly higher than the BSI depressive and anxiety symptom scores reported in a previous study with African American breast cancer survivors (ie, 3.42 and 5.38 for BSI depressive and anxiety symptom scores, respectively). ${ }^{40}$ Generally, the small body of literature indicates that Asian American breast cancer survivors experience moderate to high levels of depressive symptoms. ${ }^{14} 163133$ Psychopathology symptoms appear to be influenced by levels of acculturation, such that acculturation to mainstream US culture was associated with lower depressive symptoms ${ }^{14}$ and anxiety symptoms among Asian American breast cancer survivors. ${ }^{16}$ This finding is consistent with the broader acculturation literature that have found a link between higher levels of acculturation and lower 
levels of psychopathology symptoms. ${ }^{41}$ For instance, highly acculturated Asian American women reported lower levels of depressive symptoms than their less acculturated counterparts. ${ }^{42}$ Asian American breast cancer survivors have been found to experience a lower quality of life than European American breast cancer survivors. ${ }^{6}$ However, broad conclusions about whether Asian American breast cancer survivors experience greater levels of psychopathology than other cultural groups are difficult to draw, given the sparse number of studies that have examined multiple groups within the same study.

The second question sought to understand how various social, cognitive and affective factors are associated with psychopathology symptoms among Asian American breast cancer survivors. Studies noted that Asian cultural beliefs and norms have powerful influences on the subjective construal of social constraints. In the collectivistic relationship context, Asian individuals may be less willing to disclose their emotions or explicitly ask for support due to the shared cultural understanding that people should anticipate each other's needs for support. ${ }^{10}$ Supportseeking behaviour, such as expressing negative emotions, may lead to caregiver burden by indirectly communicating the message that they have failed to anticipate the cancer survivor's needs. While these quantitative findings support intrusive thoughts and AEE as significant mediators of the link between social constraint and psychopathology, other cognitive and emotional processes that have been highlighted in qualitative studies, such as caregiver burden and self-stigma, ${ }^{10}{ }^{25}$ remain to be tested in future research. Furthermore, Asian American breast cancer survivors' access to resources (eg, access to cancer support groups) and self-efficacy may be important moderators of the link between social constraint and psychopathology. For instance, high self-efficacy may buffer the detrimental effects of social constraints on psychological well-being.

Lastly, the third question reviewed psychosocial interventions for Asian American breast cancer survivors. Both intervention studies included in this review incorporated community-based participatory research approaches by integrating assistance and input from community organisations. For example, the researchers partnered with community organisations to identify optimal strategies for recruiting, engaging and retaining the participants in the intervention trials. The $90 \%$ and $79 \%$ retention rate reported in the expressive writing and peer-mentoring interventions, respectively, ${ }^{27}$ are impressive given the challenges that exist in recruiting Asian American breast cancer survivors in research. ${ }^{31}$ With the aid of peer mentors who are also Chinese American breast cancer survivors themselves, the 10-week education intervention programme led to reductions in anxiety and depressive symptoms. The peer mentors provided culturally sensitive and linguistic-appropriate education to help participants overcome stigmatised beliefs about cancer. ${ }^{28}$ The pilot expressive writing intervention led to reductions in post-traumatic stress symptoms and intrusive thoughts. ${ }^{27}$ However, the efficacy of these interventions should be considered cautiously, because they were both single-arm intervention trials that did not include a control condition or random assignment to conditions. Having stated that, more recent randomised controlled trials of expressive writing provide additional support for the efficacy of expressive writing interventions for Chinese American breast cancer survivors, ${ }^{43}$ but not all expressive writing interventions have led to improvements in functioning $^{44}{ }^{45}$ (Gallagher et al 2018 was not included in the review because it was published after our search ended in November 2018). Thus, more research is needed to understand the factors, such as specific writing conditions and intervening time (eg, during treatment vs during survivorship), that may explicate the mixed findings for expressive writing interventions. Generally, more intervention research with rigorous methodology designs (eg, randomised controlled trials) are needed for this population.

\section{Clinical implications}

The findings from the present review point to a number of avenues for providing culturally sensitive psychosocial care for Asian American breast cancer survivors. First, medical providers who work with this population should be educated about Asian cultural values of emotional restraint and deference to authority. As such, medical providers should provide ample opportunities for questions and be willing to repeat relevant medical information when treating Asian American breast cancer survivors, as they are less likely to verbalise their distress or confusion in fear of 'wasting' the provider's time or burdening their family members. ${ }^{26}$ Likewise, it is important to enhance medical providers' ability to accurately assess the level of psychopathology symptoms experienced by this population by educating them about their tendency to suppress their cancer-related distress. Providing Asian American breast cancer survivors with adequate mental health resources and care is critical, as they are more likely than their non-Hispanic White counterparts to experience cancer stigma and have fewer opportunities to process their cancer-related distress.

\section{Limitations and future directions}

This systematic review is the first to our knowledge to focus on the experience of psychopathology among Asian American breast cancer survivors. Although this literature has grown substantially over the past 5 years, significant gaps remain. One limitation is the lack of data on clinical levels of depression, anxiety and post-traumatic stress disorder collected through a diagnostic interview (eg, SCID-IV). Instead of a diagnostic interview, each study included in this review used a self-report (eg, Impact of Event Scale ${ }^{46}$ Center for Epidemiologic Studies Depression Scale ${ }^{47-50}$ ) or interviewer-assisted symptom checklist (eg, Edmonton Symptom Assessment System ${ }^{51}$ ). Although these instruments provide cut-off scores that can be used to identify clinical levels of psychopathology, the validity of these questionnaires to discriminate between clinical 
and non-clinical levels of psychopathology among Asian American breast cancer survivors remains to be tested. Future research can use diagnostic interviews to provide a more precise understanding of the severity of psychopathology experienced by Asian American breast cancer survivors.

Caution is needed in generalising the conclusions drawn from this review because they come from a relatively small number of participants. Indeed, seven studies included in this review utilised the same three datasets. Furthermore, the majority of the studies sampled participants from large urban cities such as San Francisco, Los Angeles and New York. The studies mostly focused on Chinese Americans and, to a lesser extent, Korean Americans. Research on the psychopathology experienced by other Asian American subgroups (eg, Vietnamese Americans, Filipino Americans, South Asian Americans) and those who live in rural regions of the USA are needed. Lastly, only two intervention studies investigated the change in psychopathology symptoms over time. More research that utilises longitudinal designs is needed to understand how social constraints impact cognitive and affective processes and their subsequent implications on psychopathology over time. Clarifying the prospective relationships among culture, social constraints and psychopathology will help inform future research and development of culturally sensitive interventions for reducing psychopathology symptoms among Asian American breast cancer survivors.

While this review highlights the small body of literature and gaps in knowledge that exist on this topic, it also reveals potentially fruitful avenues for future research. First, a large body of literature suggests that neighborhood-level factors, such as ethnic density, are associated with cancer risk and stage at diagnosis. ${ }^{52}$ However, whether residing in an ethnic enclave serves as a protective factor or vulnerability factor for psychopathology, or both, requires greater attention. Residing in an ethnic enclave may confer health benefits by preserving healthy behaviours (eg, diet) and access to healthy foods. ${ }^{53}$ Conversely, residing in an ethnic enclave may lead to greater adherence to traditional cultural beliefs and norms, such as stigmatised beliefs about cancer and limited support-seeking behaviours. Second, a nuanced examination of culture, beyond levels of acculturation, is critically needed in this literature. What specific cognitive, affective or social processes change as a result of acculturation to mainstream US culture? How do these processes interact or change among those who are highly enculturated (ie, high levels of acculturation to mainstream culture and retention of high levels of heritage culture)? For example, do highly acculturated Asian American breast cancer survivors worry less about caregiver burden when they express their cancer-related distress? Does the endorsement of stigmatised beliefs about cancer, such as the belief that cancer is caused by one's misdeeds, decrease as a result of exposure to Western media and social interactions with European
Americans? Both qualitative and quantitative methodologies are needed to unpack 'what' and 'how' cultural factors influence social constraints and their implications for psychopathology among this population.

Contributors WT designed the study. SN and RZ conducted the search, data quality assessment and coding of key study information. WT wrote the manuscript with critical feedback from SN and RZ.

Funding The authors have not declared a specific grant for this research from any funding agency in the public, commercial or not-for-profit sectors.

Competing interests None declared.

Patient and public involvement Patients and/or the public were not involved in the design, or conduct, or reporting or dissemination plans of this research.

Patient consent for publication Not required.

Provenance and peer review Not commissioned; externally peer reviewed.

Data availability statement Data sharing not applicable as no datasets generated and/or analysed for this study. No dataset was used in this systematic review.

Open access This is an open access article distributed in accordance with the Creative Commons Attribution Non Commercial (CC BY-NC 4.0) license, which permits others to distribute, remix, adapt, build upon this work non-commercially, and license their derivative works on different terms, provided the original work is properly cited, appropriate credit is given, any changes made indicated, and the use is non-commercial. See: http://creativecommons.org/licenses/by-nc/4.0/.

ORCID iD

William Tsai http://orcid.org/0000-0002-8889-0575

\section{REFERENCES}

1 Torre LA, Sauer AMG, Chen MS, et al. Cancer statistics for Asian Americans, Native Hawaiians, and Pacific Islanders, 2016: converging incidence in males and females. CA Cancer J Clin 2016;66:182-202.

2 Colby SL, Ortman JM. Projections of the size and composition of the US population: 2014 to 2060. US Census Briefs, 2015.

3 Gomez SL, Clarke CA, Shema SJ, et al. Disparities in breast cancer survival among Asian women by ethnicity and immigrant status: a population-based study. Am J Public Health 2010;100:861-9.

4 Gomez SL, Von Behren J, McKinley M, et al. Breast cancer in Asian Americans in California, 1988-2013: increasing incidence trends and recent data on breast cancer subtypes. Breast Cancer Res Treat 2017;164:139-47.

5 Siegel RL, Miller KD, Jemal A. Cancer statistics, 2019. CA Cancer J Clin 2019;69:7-34.

6 Wen K-Y, Fang CY, Ma GX. Breast cancer experience and survivorship among Asian Americans: a systematic review. J Cancer Surviv 2014;8:94-107.

7 Fann JR, Thomas-Rich AM, Katon WJ, et al. Major depression after breast cancer: a review of epidemiology and treatment. Gen Hosp Psychiatry 2008;30:112-26.

8 Robson A, Scrutton F, Wilkinson L, et al. The risk of suicide in cancer patients: a review of the literature. Psychooncology 2010;19:1250-8.

9 Anguiano L, Mayer DK, Piven ML, et al. A literature review of suicide in cancer patients. Cancer Nurs 2012;35:E14-26.

10 Wong-Kim E, Sun A, Merighi JR, et al. Understanding qualityof-life issues in Chinese women with breast cancer: a qualitative investigation. Cancer Control 2005;12:6-12.

11 Hsu BY, Chentsova Dutton Y, Adams IF, et al. Talking about cancer: explaining differences in social support among Chinese American and European American breast cancer survivors. J Health Psychol 2020;25:1043-56.

12 Markus HR, Kitayama S. Culture and the self: implications for cognition, emotion, and motivation. Psychol Rev 1991;98:224-53.

13 Bond $\mathrm{MH}$. Emotions and their expression in Chinese culture. $J$ Nonverbal Behav 1993;17:245-62.

14 Lim J-won, Yi J, Zebrack B. Acculturation, social support, and quality of life for Korean immigrant breast and gynecological cancer survivors. Ethn Health 2008;13:243-60.

15 Wu IHC, McNeill LH, Lu Q. Ambivalence over emotional expression and physical functioning and limitations: mediating and moderating effects of PTSD symptoms and acculturation among Chinese breast cancer survivors. Support Care Cancer 2019;27:1-9. 
16 Yi JK, Swartz MD, Reyes-Gibby CC. English proficiency, symptoms, and quality of life in Vietnamese- and Chinese-American breast cancer survivors. J Pain Symptom Manage 2011;42:83-92.

17 Moher D, Liberati A, Tetzlaff J, et al. PRISMA - Review guidelines. Ann Intern Med 2009.

18 Stroup DF, Berlin JA, Morton SC, et al. Meta-Analysis of observational studies in epidemiology: a proposal for reporting. meta-analysis of observational studies in epidemiology (MOOSE) group. JAMA 2000;283:2008-12.

19 Lepore SJ. A social-cognitive processing model of emotional adjustment to cancer [online]. Psychosocial interventions for cancer. Washington, DC: American Psychological Association, 2001: 99-116.

20 Lepore SJ, Helgeson VS. Social constraints, Intrusive thoughts, and mental health after prostate cancer. J Soc Clin Psychol 1998;17:89-106.

21 Roberts KJ, Lepore SJ, Helgeson V. Social-cognitive correlates of adjustment to prostate cancer. Psychooncology 2006;15:183-92.

22 Husson O, Mols F, van de Poll-Franse LV. The relation between information provision and health-related quality of life, anxiety and depression among cancer survivors: a systematic review. Ann Oncol 2011;22:761-72.

23 Mols F, Vingerhoets AJJM, Coebergh JW, et al. Quality of life among long-term breast cancer survivors: a systematic review. Eur J Cancer 2005;41:2613-9.

24 Ashing-Giwa KT, Padilla G, Tejero J, et al. Understanding the breast cancer experience of women: a qualitative study of African American, Asian American, Latina and Caucasian cancer survivors. Psychooncology 2004;13:408-28.

25 Lee S, Chen L, Ma GX, et al. Challenges and needs of Chinese and Korean American breast cancer survivors: in-depth interviews. N Am J Med Sci 2013:6:1-8.

26 Kagawa-Singer M, Wellisch DK, Durvasula R. Impact of breast cancer on Asian American and Anglo American women. Cult Med Psychiatry 1997;21:449-80.

27 Lu Q, Zheng D, Young L, et al. A pilot study of expressive writing intervention among Chinese-speaking breast cancer survivors. Health Psychol 2012;31:548-51.

28 Lu Q, You J, Man J, et al. Evaluating a culturally tailored peermentoring and education pilot intervention among Chinese breast cancer survivors using a mixed-methods approach. Oncol Nurs Forum 2014;41:629-37.

29 Vin-Raviv N, Hillyer GC, Hershman DL, et al. Racial disparities in posttraumatic stress after diagnosis of localized breast cancer: the BQUAL study. J Natl Cancer Inst 2013;105:563-72.

30 Lu Q, Man J, You J, et al. The link between ambivalence over emotional expression and depressive symptoms among Chinese breast cancer survivors. J Psychosom Res 2015;79:153-8.

31 Paek M-S, Lim J-W, won LJ. Understanding the stress process of Chinese- and Korean-American breast cancer survivors. J Immigr Minor Health 2016;18:1159-67.

32 Tsai W, Lu Q. Acculturation matters in the relation between ambivalence over emotional expressions and well-being among Chinese American breast cancer survivors. Qual Life Res 2017;26:2755-62.

33 Gonzalez BD, Lu Q. Sleep disturbance among Chinese breast cancer survivors living in the USA. Support Care Cancer 2018;26:1695-8.

34 Wong CCY, Warmoth K, Ivy S, et al. Relation of social constraints on disclosure to adjustment among Chinese American cancer survivors: a multiprocesses approach. Psychooncology 2018;27:977-82.

35 Lu Q, Yeung N, Man J, et al. Ambivalence over emotional expression, intrusive thoughts, and posttraumatic stress symptoms among Chinese American breast cancer survivors. Support Care Cancer 2017;25:3281-7.

$36 \mathrm{Lim}$ J-W, Lim J. The impact of comorbidity on the relationship between life stress and health-related quality of life for Chinese- and Korean-American breast cancer survivors. Ethn Health 2018;23:16-32.

37 Wohlfarth TD, van den Brink W, Winkel FW, et al. Screening for posttraumatic stress disorder: an evaluation of two self-report scales among crime victims. Psychol Assess 2003;15:101-9.

38 King LA, Emmons RA. Conflict over emotional expression: psychological and physical correlates. J Pers Soc Psychol 1990;58:864-77

39 Morrill EF, Brewer NT, O'Neill SC, et al. The interaction of posttraumatic growth and post-traumatic stress symptoms in predicting depressive symptoms and quality of life. Psychooncology 2008;17:948-53.

40 Schover LR, Jenkins R, Sui D, et al. Randomized trial of peer counseling on reproductive health in African American breast cancer survivors. J Clin Oncol 2006;24:1620-6.

41 Suinn RM. Reviewing acculturation and Asian Americans: how acculturation affects health, adjustment, school achievement, and counseling. Asian Am J Psychol 2010;1:5-17.

42 Hung $\mathrm{JH}$. The predictive ability of acculturation with psychological adjustment and gender role attitudes among Asian American women. Dissertation Abstracts international: section B: the sciences and engineering, 2007.

43 Lu Q, Gallagher MW, Loh A, et al. Expressive writing intervention improves quality of life among Chinese-American breast cancer survivors: a randomized controlled trial. Ann Behav Med 2018;52:952-62.

44 Lu Q, Wong CCY, Gallagher MW, et al. Expressive writing among Chinese American breast cancer survivors: a randomized controlled trial. Health Psychol 2017;36:370-9.

45 Gallagher MW, Long LJ, Tsai W, et al. The unexpected impact of expressive writing on posttraumatic stress and growth in Chinese American breast cancer survivors. J Clin Psychol 2018;74:1673-86.

46 Falsetti SA, Resnick HS, Resick PA, et al. The modified PTSD symptom scale: a brief self-report measure of posttraumatic stress disorder. Behav Ther 1993

47 Radloff LS. The CES-D scale: a self-report depression scale for research in the general population. Appl Psychol Meas 1977.

48 Derogatis LR, Fitzpatrick M. The SCL-90-R, the Brief Symptom Inventory (BSI), and the BSI-18. In: The use of psychological testing for treatment planning and outcomes assessment: volume 3 : instruments for adults, 2004.

49 Sundin EC, Horowitz MJ. Impact of event scale: psychometric properties. Br J Psychiatry 2002;180:205-9.

50 McHorney CA, Ware JE, Raczek AE. The MOS 36-Item short-form health survey (SF-36): II. psychometric and clinical tests of validity in measuring physical and mental health constructs. Med Care 1993:31:247-63.

51 Bruera E, Kuehn N, Miller MJ, et al. The Edmonton symptom assessment system (ESAs): a simple method for the assessment of palliative care patients. J Palliat Care 1991;7:6-9.

52 Fang CY. Ethnic density and cancer : a review of the evidence, 2018

53 Osypuk TL, Diez Roux AV, Hadley C, et al. Are immigrant enclaves healthy places to live? the multi-ethnic study of atherosclerosis. Soc Sci Med 2009;69:110-20.

54 Suinn RM, Rickard-Figueroa K, Lew S, et al. The Suinn-Lew Asian self-identity acculturation scale: an initial report. Educ Psychol Meas 1987;47:401-7

55 Chung RHG, Kim BSK, Abreu JM. Asian American multidimensional acculturation scale: development, factor analysis, reliability, and validity. Cultur Divers Ethnic Minor Psychol 2004;10:66.

56 Stephenson M. Development and validation of the Stephenson Multigroup Acculturation scale (SMAS). Psychol Assess 2000;12:77-88 